\title{
Electron Beam Ablation Phenomenon - Theoretical Model and Applications
}

\author{
V.E. Ptitsin \\ Additional information is available at the end of the chapter
}

http://dx.doi.org/10.5772/47824

\section{Introduction}

The term "ablation" (i.e., removal or disposal of material) is quite often used in publications devoted to studies on physical and physicochemical processes caused by interactions of concentrated energy fluxes with absorbing condensed substance.

Most of the works on ablation relate to investigation or practical application of processes developed due to condensed matter interaction with high-power laser beams. In fact, now laser ablation is widely used in time-of-flight mass spectrometry as well as in modern microand nanoelectronics and microelectromechanics for structural surface modification and profiling of condensed substances.

However despite considerable academic and practical interest aroused by the ablation phenomenon, so far it has not been given a commonly adopted and (or) unique definition.

This may be so because of a too broad meaning put into the term ablation. Generally it means both the removal (disposal) of substance in the form of its certain fragments (droplets, macromolecules, clusters) under different physical conditions and fast phase transition of atoms and molecules of condensed matter from bound to free (excited or ionized) states. In such extended interpretation, this term seems to present no heuristic value.

In view of the aforesaid, in the present work the phenomenon of ablation is treated in a narrower physical sense, namely as a fast process of phase bound-free transition in excited or ionized structural elements (atoms, molecules) of condensed substance exposed to intense corpuscular radiation, e.g., intense electron fluxes.

It is known that interaction of a substance with intense either laser or electron beams exhibits some common regular features which have not yet been interpreted unambiguously. These are the following [1-5]. 
1. The phenomena of laser ablation and EBA are of threshold nature, i.e., they appear only when the beam intensity or the electron flux power density $(P)$ exceeds some threshold value $\left(P_{1}\right)$ characteristic of a given substance. For EBA in a wide range of condensed media, $P_{1} \approx 50 \mathrm{MW} / \mathrm{cm}^{2}$.

2. There is a finite time delay $(\Delta t)$ between the onset of the substance exposure to a concentrated energy flux and the moment of phase transition of the substance to plasma. The time $\Delta t$ strongly depends on the $P$ level and can widely vary from $\approx 10^{-8} \mathrm{~s}$ $\left(P \geq P_{1}\right)$ to $\approx 10^{-13} \mathrm{~s}$ (at $\left.P>>P_{1}\right)$.

3. The charge content formed during dense plasma ablation also substantially depends on $P$. At the initial stage of plasma formation (at $P$ close to $P_{1}\left(P>P_{1}\right)$ native excited atoms (molecules) of a substance remain mostly neutral when going from bound to the free states. At high levels of $P\left(P>>P_{1}\right)$ the average charge number $(Z)$ of plasma ions may be $Z \approx(2-3)$.

The purpose of the present part of the work is to study the physical mechanism of the above features. In the following sections of the paper we offer a phenomenological model of the EBA and interpretation of the two characteristic features of this phenomenon.

\section{Theoretical model of the EBA phenomenon}

\subsection{Heat conducting as a limiting factor for the rate of energy absorption by a condensed substance}

To calculate the rate of incident radiation energy absorption by a condensed substance (in units of $\mathrm{W} / \mathrm{m}^{2}$ ), we consider the condensed substance as a set of two interrelated subsystems: those of electrons and phonons.

It is known that when the substance surface is exposed to electromagnetic radiation or electron flux, the incident flux energy is mainly absorbed by the electron subsystem and then, due to electron-phonon interaction, is transferred to phonon subsystem. The energy transferred to the phonon subsystem propagates in the substance through the phononphonon interaction or the mechanism of heat conduction.

Based the above scheme of radiation energy absorption by the substance, we calculate estimates for the heat conduction flux power density $(\Theta)$ existing in this case. In a onedimensional approximation, the heat flux propagating from the surface into the bulk of the substance, $\Theta$ can be expressed as [6]

$$
\Theta \approx \frac{1}{3} \cdot n_{f} \cdot V_{s} \cdot \varepsilon
$$

where $n_{f}$ is the phonon concentration, $V_{s}$ is the sound velocity, $\varepsilon$ is the average energy transferred by a phonon at its transition with a local temperature $T+\Delta T$ to the region with the temperature $T$. In Debye's approximation, the average phonon energy is defined by the known expression 


$$
\varepsilon \cong 9 \cdot \frac{N}{n_{f}} \cdot k \theta \cdot \xi^{4} \cdot \int_{o}^{1 / \xi} d x \cdot \frac{x^{2}}{e^{x}-1},
$$

where $N$ is the concentration of atoms in the substance, $k$ is the Boltzmann constant, $\theta$ is the Debye temperature, $\xi \equiv T / \theta$. Integrating (2) in view of (1) yields

$$
\Theta \cong N k \theta V_{s} \xi\left[1-\frac{3}{8 \xi}+3 \sum_{n=1}^{\infty} \frac{(-1)^{n+1} B_{n} \xi^{-2 n}}{(2 n) ! \cdot(2 n+3)}\right]
$$

where $B_{n}$ are Bernoulli's numbers.

The analysis of the series in (3) shows its convergence, so if $\xi \geq 1$, Eq. (3) can be written in a more compact form:

$$
\Theta \approx \gamma \cdot N \cdot k \theta \cdot V_{s}
$$

where $\gamma=\gamma(\xi)$ is a dimensionless parameter whose numerical value is close to $\gamma \sim 0.5$ at $\xi \approx 1$. Eq. (4) shows that $\Theta$ only weakly depends on the substance nature since the respective substance characteristics present in (4) relatively little vary from substance to substance. Substituting the parameter values into (4) gives the value of $\Theta$ for a wide range of substances: $\Theta \approx(50 \pm 20) \mathrm{MW} / \mathrm{cm}^{2}$.

Thus the obtained estimates of $\Theta$ and empirical values of $P_{1}$ suggest that the existing ablation threshold results from the finiteness of the dissipation rate (in the phonon subsystem) of the radiation energy absorbed by the substance.

Besides, from the obtained estimate and the energy conservation law it follows that if the energy input velocity of the radiation incident on a condensed substance exceeds $\Theta$, then the energy of the incident radiation (or electron flux) must be "reflected" by the substance in some way or another.

In the next section we obtain expressions for the ablation threshold $P_{1}$ and delay time $\Delta t\left(P_{l}, P\right)$ by the example of interaction of an intense electron flux with metals. It is also shown that at $P>$ $P_{1}$ the reflection of a concentrated energy flux by a substance may result from a fast phase transition of atoms (molecules) in the condensed substance from bound to free excited and ionized states. It seems evident that the physical "carrier" of the reflected energy flux during ablation is a flow of excited neutrals and ions of the condensed substance.

\subsection{Modeling of the ablation process in the metal exposed to a concentrated energy flux - High-power density electron beam}

To build the model of ablation, it would be natural to assume that the physical reason for the phase bound-free transition of surface excited or ionized atoms is that the binding (or cohesion) energy of atoms is reduced due to the excitation of the electron subsystem layer by external radiation or a high intensity corpuscular flux. 
To build a model for the mechanism of processes occurring in a substance when the beam power density exceeds the level $(\Theta)$ which can be absorb through the mechanism of thermal conduction we consider the interaction of an intense electron flux with the metal surface. Note that such choice of the energy "carriers" and the "object" exposed to the energy flux is made to simplify description and analysis of the interaction of a concentrated energy flux with the substance.

The interaction between the electron and phonon subsystems is described based on the well known relaxation time approximation $[3,6]$.

Using the concepts developed in [6], the absolute value of binding energy at the excitation of the electron subsystem in the metal $\left(\Lambda^{*}\right)$ can be defined as

$$
\Lambda^{*} \approx \Lambda-r \cdot\left(\mu-\frac{3}{5} \cdot E_{f}\right)
$$

where $\Lambda$ is the absolute value of the surface atom binding energy in the absence of electron subsystem excitation, $r(r \approx 0.6-0.7)$ is the dimensionless coefficient equal (in absence of electron subsystem excitation) to the average binding to cohesion energy ratio of the surface atom, $\mu$ is the average kinetic energy of the conducting electron, $E_{f}$ is the Fermi energy. In the free electron model for metals the balance equation for the conducting electron kinetic energy $\mu$ in the metal exposed to an electron flux in a one-dimensional approximation can be written as [7-9]

$$
\frac{d \mu}{d t}=\frac{(1-\eta) \cdot P \cdot \varphi(E)}{n \cdot E}-\frac{\varepsilon}{2 \tau} \cdot \frac{k \cdot\left(T_{e}-T_{p}\right)}{E_{f}},
$$

where $\eta$ is a dimensionless coefficient characterizing elastic electron scattering by the metal surface and also non-zero probability of emissive recombination of excited electrons (according to [3], $\eta<0.1), E$ is the energy of electron striking the metal surface, $\varphi(E)$ is the specific loss function of an electron injected into the metal subsurface layer; note that the $\varphi(E)$ value for the metal "surface" points should be calculated from the known Bethe formula for inelastic energy losses (see, e.g., [3]); besides, since Bethe's formula is approximate, in quantitative calculations, the theoretical value of this function needs correction based on the known experimental data; $n$ is the conduction electron concentration in metal; $P$ is the electron flux power density; $\tau$ is the characteristic time of electron-phonon interaction; $T_{p}$ are the absolute electron and lattice temperatures, respectively.

Generally, integrating Eq. (6) requires specifying and finding the function $T_{p} \equiv T_{p}(t)$. However, as shown in [2], when the metal is exposed to intense electron fluxes, during the delay time (about $\approx 10^{-8} \mathrm{~s}$ ) the lattice temperature increases only slightly and for, e.g., $\mathrm{Cu}$ it does not exceed $\approx 800 \mathrm{~K}$. In this connection and taking into account that the heat capacity of a metal lattice is much greater than that of the electron gas, when integrating Eq. (5), one can assume $T_{e} \gg T_{p}=$ const. The relationship between $T_{e}$ and $\mu$ is of the form [10] 


$$
k T_{e} \approx\left[\mu^{2}-\left(\frac{3}{5} \cdot E_{f}\right)^{2}\right]^{1 / 2}
$$

Using for Eq. (7) the approximation

$$
k T_{e} \approx 3 \cdot\left(\mu-\frac{3}{5} E_{f}\right),
$$

with a maximum error of $20 \%$ in the interval $0.6 E_{f} \leq \mu \leq E_{f}$, from Eqs. (5-8) with the initial condition $\mu(0)=0.6 E_{f}$, we obtain

$$
\Lambda^{*}(P, t) \approx \Lambda-\frac{2 r \cdot(1-\eta) \cdot \tau E_{f} \varphi P}{3 \cdot \varepsilon \cdot n \cdot E} \times\left[1-\exp \left(-\frac{3 \varepsilon}{2 E_{f}} \cdot \frac{t}{\tau}\right)\right]
$$

Setting $\Lambda^{*}(P, t)$ equal to zero and assuming $t \gg \tau$, from (9) one can find an expression for the minimum (or threshold) value of electron flux power density $\left(P_{1}\right)$ which starts the excitation of the metal electron subsystem accompanied by the decrease in surface atom binding energy (down to zero). In other words, the conditions $\Lambda^{*}(P, t)=0, t>\tau$ physically mean that at $P \geq P_{1}$ after a certain time interval (delay time), "pumping" of the electron subsystem leads to a bound-free transition of excited and (or) ionized metal surface atoms not held by binding energies.

According to (9), the threshold level of power density can be expressed as

$$
P_{l} \approx \frac{3 \cdot \varepsilon \cdot n \cdot E \cdot \Lambda}{2 \cdot r \cdot(1-\eta) \cdot \tau \cdot E_{f} \cdot \varphi}
$$

Using the numerical estimates of from (10) for $\mathrm{Cu}$, obtain

$$
P_{l} \approx \Theta
$$

The delay time $\Delta t$ (at $P>P_{1}$ ) can be also calculated from Eq. (9). After some rearrangements from (9) we have

$$
\Delta t \approx \frac{-2}{3} \cdot \frac{E_{f}}{\varepsilon} \cdot \tau \cdot \ln \left(1-\frac{P_{l}}{P}\right)
$$

The calculations of (12) show that in agreement with experimental data [1-4] $\Delta t$ strongly depends on $P$ and (for currently realized $P$ levels) may vary from $\approx\left(10^{-7}-10^{-8}\right)$ s to $\approx 10^{-13} \mathrm{~s}$.

To conclude, we estimate the velocity of motion of the condensed substance-plasma boundary $(v)$ in the course of ablation process. In the framework of the proposed model of ablation the activation energy value for the event of atomic bound-free transition can be assumed to be zero. 
Based on the known concepts of Arrhenius-Frenkel about the probabilistic character of thermally activated bound-free transitions of surface atoms, the inter phase boundary motion velocity during the process of ablation can be expressed in the form of

$$
v \approx \lim _{\Lambda^{*} \rightarrow 0}\left[a f \cdot \exp \left(-\frac{\Lambda^{*}}{k T_{p}}\right)\right]=a \cdot \frac{k T_{p}}{h},
$$

where $a$ is the lattice parameter,

$$
f \approx \frac{k T_{p}}{h}
$$

is the surface atom oscillation frequency. For example, for $\mathrm{Cu}$ one can easily access from (13) that already at a temperature $T_{p}$ close to $\approx 500 \mathrm{~K}$, the velocity $v$ reaches values $\approx V_{s}$. The estimates obtained for $v$ and $T_{p}$ are in quite satisfactory quantitative agreement with the data of $[1,2]$. Eq. (13) also shows that during the process of ablation the ionic subsystem of a substance cannot heat substantially since the thermal front velocity in substance, in Debye's approximation does not exceed $V_{s}$. Besides, note that the proportionality between $v$ and $T_{p}$ and also the finiteness of the thermal front velocity must lead to mutual "selfconsistency" of the velocities $v$ and $V_{s}$ and, hence, to their equalization during ablation.

Note that the inference made above about the relatively low temperature of the ion subsystem in the process of ablation does not contradict the known fact that ablation results in erosion markings and surface-scarred craters formed at the substance surface [1-4].

As consistent with the proposed ablation model, considerable heating and fusing of the substance may take place when $v<V_{s}$ and power density of the concentrated energy flux at the substance surface is less than $P_{1}$, accordingly. First of all such decrease in power density would always happen at the trailing edge of the energy pulse (down to $1 \mathrm{~ns}$ and less) high-power pulses are usually "bell-shaped". Second, if the energy pulse is relatively long, the level of the power density absorbed by the substance can fall to the values $P<P_{1}$ in a substantially shorter time than the pulse duration due to the growth of plasma thickness and density with time and, hence, of the efficiency of energy absorption in plasma.

Note, finally, that termination of the energy pulse action (irrespective of the pulse shape and width) and falling of $P$ to $P<P_{1}$ would always be accompanied by the ion subsystem temperature rise over that in the ablation mode. This is because at $P<P_{1}$ the ionic subsystem of the subsurface layer will be heated as a result of both plasmoid disintegration and inertial absorption of the electron excitation energy by the ionic subsystem.

Thus, the EBA phenomenon is investigated on the basis of the general thermodynamic representations about power balance of absorption and dissipation energy processes in conditions of interaction of the concentrated energy flux with a condensed substance. It is shown that at high levels of absorbed power density, exceeding energy dissipation speed by the heat conductivity mechanism, the fast phase transition of a condensed substance in 
dense plasma can take place. The offered and developed general representations about the physical mechanism of the EBA phenomenon [11] allow suggest adequate interpretation (as qualitatively and quantitatively) of the basic laws of this phenomenon.

\section{EBA phenomenon applications}

In the recent two decades EBA phenomenon finds use in different areas of science and technologies [5, 9-20]. At the present time EBA is considered as one of alternatives to pulsed laser ablation (LA) which is more advanced. EBA possesses a number of important advantages: low-cost equipment (5-10\% of the laser systems), big efficiency, possibility of ablation of optically transparent materials (e.g., $\mathrm{SiO}_{2}$ ) and some others $[12,13]$. EBA (or super-fast phase transition of solids into dense plasma plume) is one of the promising applications of high-intensive (more than $\left.10 \mathrm{kA} / \mathrm{cm}^{2}\right)$, low-energy $(5-10 \mathrm{keV})$ pulsed ( 100 $\mathrm{ns}$ ) electron beams of small diameter (from $\sim 1 \mathrm{~nm}$ to $\sim 10^{6} \mathrm{~nm}$ and more).

By their physical nature the phenomena of LA and EBA are caused by the interaction of the concentrated energy fluxes with the condensed substance.

Of course the physical mechanisms of excitation and formation of plasma plumes for the processes LA and EBA have their special features; however, phenomenology of both processes, and in particular, the plasma density and also the dynamics of formation and dispersion of plasmoid (plume) are sufficiently close [5].

In connection with this the circle of scientific and technological tasks and problems, which can be solved on the basis of the use of the EBA phenomenon, to a considerable extent coincides with the spectrum of the tasks, solved at present by the LA method.

As it follows from the previous examination, EBA process is excited only when the electron beam power density $P$ exceeds $P_{1}$

$$
\mathrm{P}=\left(\frac{1}{e S_{b}}\right) \cdot I_{b} \cdot E_{b} \geq P_{l} \cong 50 \mathrm{MW} / \mathrm{cm}^{2},
$$

where e is the electron charge, $S_{b}$ is the cross section of the beam on a substance (target) surface, $I_{b}$ is the beam current, $E_{b}(\mathrm{eV})$ is the beam energy on a substance (target) surface. Depending on the formulation of the scientific or technological problem the condition for the excitation EBA (14) can be realized by the electron beams, whose parameters: a radius $\left(r_{b}\right)$ of beam, $I_{b}, E_{b}$ can vary over wide limits.

Among the pioneer works on EBA study it is possible to note work [16]. For conducting of studies EBA phenomenon were used the pulsed ( $100 \mathrm{~ns})$ electron beams of small diameter (approximately of $1 \mathrm{~mm}$ ) with these typical parameters: beam current density $\left(J_{b}\right)$ - more than $10 \mathrm{kA} / \mathrm{cm}^{2}$, beam energy 5-10 keV. In this work the dynamics of electron beam ablation plumes $\left(\mathrm{SiO}_{2}\right)$ have been characterized through the application of dye laser resonance absorption photography. Plume front expansion velocities of $\mathrm{Si}$ atoms were measured at nearly $1 \mathrm{~cm} / \mu \mathrm{s}$, and are comparable to the expansion of laser ablated metal atom plumes with laser fluences of a few $\mathrm{J} / \mathrm{cm}^{2}$. 
For using the EBA phenomenon for the solution of the problems of micro- and the nanotechnologies is, accordingly, necessary intensive $\left(P>50 \mathrm{MW} / \mathrm{cm}^{2}\right)$ electron beams with a $r_{b}$ from $\sim 1 \mathrm{~nm}$ to $\sim 1 \mathrm{~mm}$. The formation of electron beams with such parameters is completely nontrivial task.

At the present time practical solution of this problem is obtained for the $\mathrm{rb} \approx 1 \mathrm{~mm}$ [21]. In the Neocera Company [21] are developed and in practice realized systems and installations for the formations of powerful pulsed electron beams $(\mathrm{rb} \sim 1 \mathrm{~mm})$, which permit implementation of a process of pulse electronic beam ablation. According to data of the Neocera Company process EBA is the process in which a pulsed (100 ns) high power electron beam (approximately $10^{3} \mathrm{~A}, 15 \mathrm{keV}$ ) penetrates approximately $1 \mu \mathrm{m}$ into the target resulting in a rapid evaporation of target material, and its transformation in plasma state. The non-equilibrium extraction of the target material (ablation) facilitates stochiometric composition of the plasma. Under optimum conditions, the target stoichiometry is thus preserved in the deposited films. All solid state materials-metals, semiconductors and insulators, can be deposited as thin films with EBA. As in the case of Pulsed Laser Deposition (PLD), the pulsed electron deposition (PED) technique provides a unique platform for depositing thin films of complex materials on a variety of technologically important substrates, with a unique strength of extending the range of materials and applications. Unlike PLD, where the ablation process is critically dependent on the optical absorption coefficient of the target material, in PED, the ablation depends only on the range of electrons in the target. For most of the solid state materials, this range is of the order of a few microns. For example, $\mathrm{SiO}_{2}$ with a large optical band-gap of $10 \mathrm{eV}$ is transparent to the $248 \mathrm{~nm} \mathrm{Kr}-\mathrm{F}$ excimer laser radiation. In PED technique however, the high-power electrons can strongly couple to the target material $\left(\mathrm{SiO}_{2}\right)$, leading to $\mathrm{SiO}_{2}$ film deposition. The beamsolid interaction mechanism is quite different in PED in comparison to PLD. This unique difference provides thin film experimentalists a mechanism to extend the parameter-space required for certain novel materials fabrication. Let us note also some representative materials systems explored by PED [21]:

- High-Temperature Superconducting (HTS) YBCO (and GdBCO) films.

- Metallic-oxide (SrRuO3) films.

- Paraelectric (Ba-SrTiO3) films.

- Insulating glass ( $\mathrm{SiO} 2-$ based) films and $\mathrm{Al} 2 \mathrm{O} 3$ films.

However, formation of high intensity electron beams in the so-called sub-micron range $\mathrm{rb}$ from $\sim 0.01 \mu \mathrm{m}$ to $\sim 1 \mu \mathrm{m}$ up to now are not thus far created. In this direction thus far there are only separate studies [14, 15], in which is theoretically and experimentally substantiated the possibility of designing of beams with the high power density (up to $\sim 100 \mathrm{GW} / \mathrm{cm}^{2}$ ).

Thus, for the range of $0.01 \mu \mathrm{m} \leq \mathrm{rb} \leq 1 \mu \mathrm{m}$ the problem of the creation forming systems of the electron beams, the power density of which would satisfy condition (14) as long as remains not solved. 
Considerable achievements in the region of the practical applications of the EBA phenomenon in science the nanotechnology it was possible to attain as an instrument the transmission electron microscopy (TEM) technique [17 - 20].

This based on the EBA phenomenon method [17 - 20] of the studies is used as the investigation tool a high-resolution transmission electron microscope (TEM) to fabricate various (with sub-nm resolution) nanostructures and nanodevices including nanogaps, nanodiscs, nanorings, nanochannels, nanowires with tailored curvatures and multi-terminal nanogap devices with nanoislands or nanoholes between the terminals. This method was called TEBAL (transmission electron beam ablation lithography). During TEBAL, the user is able in situ to watch on the fluorescent imaging screen of the TEM the ablation process of the unwanted metal, followed by the crystallization of the surrounding metal and the formation (Fig.1) of desired geometries [17]. The high resolution, geometrical flexibility and yield make this fabrication method unique and attractive for many applications including nanoelectronics, nanofluidics, plasmonics, nanoparticle/atom manipulation on a chip. Particularly exciting and unique are the nanogap-nanohole devices as they open new opportunities for DNA nanopore sequencing [22].
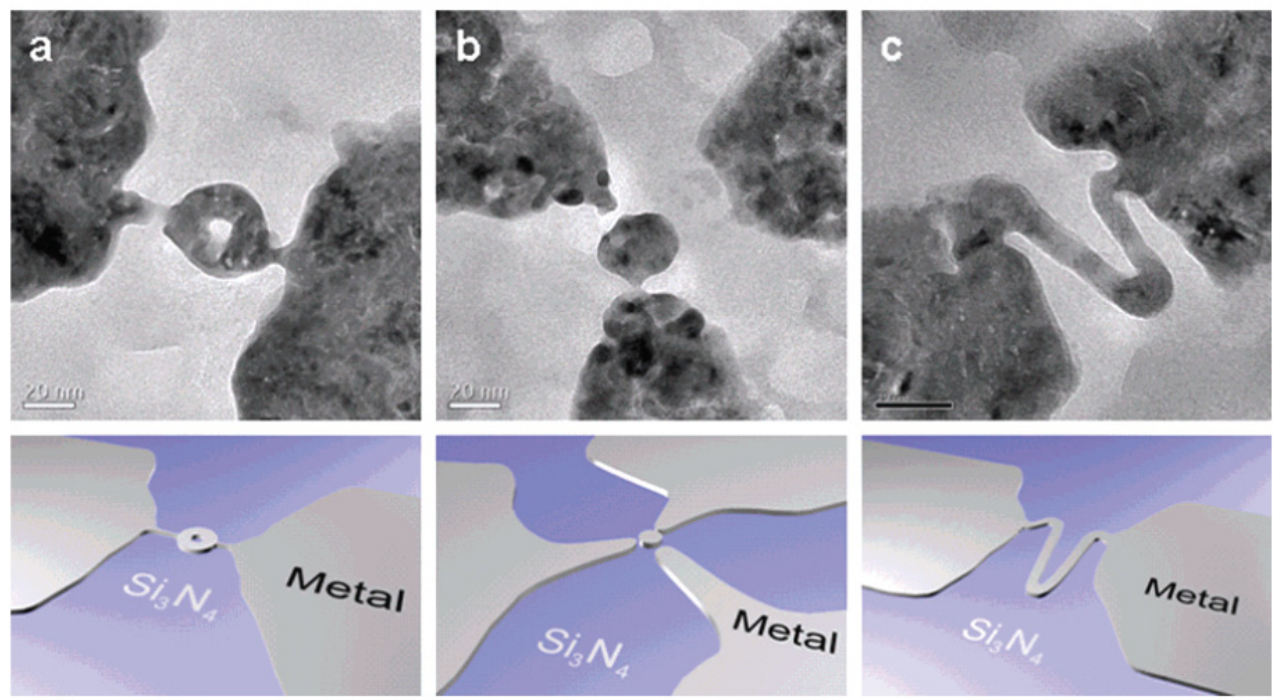

Figure 1. [17]. Example structures to demonstrate the flexibility of TEBAL. Each of the three structures shown in the TEM images is accompanied by a schematic (below) showing the fabrication by TEBAL.

(a) Nanoring with outer radius of $\sim 18.5 \mathrm{~nm}$ and inner radius of $\sim 3 \mathrm{~nm}$ (scale $=20 \mathrm{~nm}$ ). (b) Three-terminal electronic device: source and drain leads are coupled to a $\sim 13 \mathrm{~nm}$ radius metallic island and a gate electrode $\sim 23 \mathrm{~nm}$ away from the island (scale $=20 \mathrm{~nm}$ ). The rate-limiting tunneling barrier (upper junction) is a $2.7 \mathrm{~nm}$ gap. (c) Serpentine wire with $6 \mathrm{~nm}$ width (scale $=20 \mathrm{~nm}$ ). All lengths were measured with Gatan's Digital Micrograph image analysis software. 
In the recent work [20] it communicates about the electronic measurements on high quality graphene nanoconstrictions (GNCs) fabricated in a transmission electron microscope (TEM), and the first measurements on GNC conductance with an accurate measurement of constriction width down to $1 \mathrm{~nm}$. To create the GNCs, freely suspended graphene nanoribbons (GNR) were fabricated using few-layer graphene grown by chemical vapor deposition. The ribbons were loaded into the TEM, and a current-annealing procedure was used to clean the material and improve its electronic characteristics. The TEM beam was then used to sculpt GNCs to a series of desired widths in the range 1-700 nm; after each sculpting step, the sample was imaged by TEM and its electronic properties were measured in situ. The diagram of experiments [20] and some results are given in figure 2. GNC conductance was found to be remarkably high, comparable to that of exfoliated graphene samples of similar size. The GNC conductance varied with width approximately as $G(w)=$ $\left(\mathrm{e}^{2} / \mathrm{h}\right) \mathrm{w}^{0.75}$, where $\mathrm{w}$ is the constriction width in nanometers. GNCs support current densities greater than $\sim 120 \mu \mathrm{A} / \mathrm{nm}^{2}, 2$ orders of magnitude higher than that which has been previously reported for graphene nanoribbons and 2000 times higher than that reported for copper.
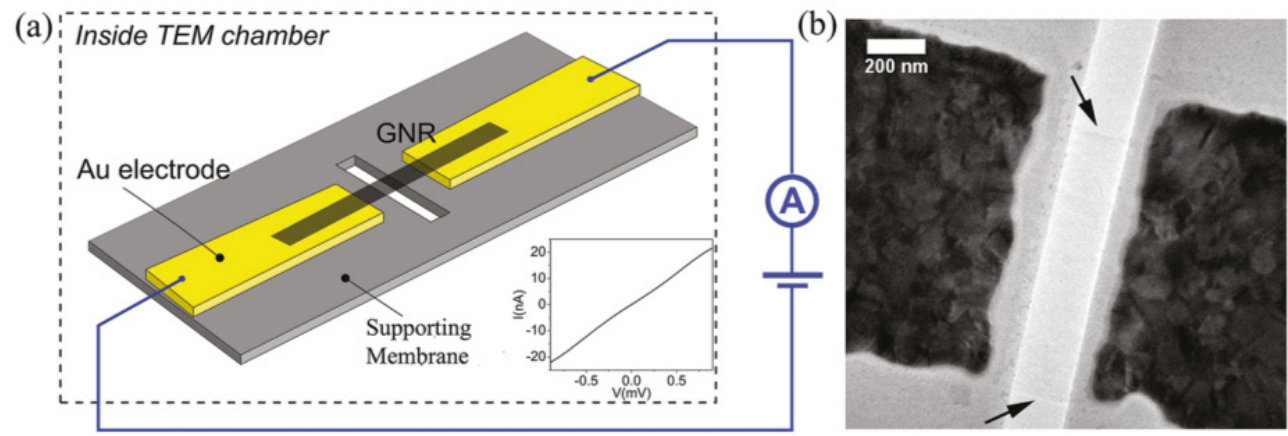

Figure 2. [20]. Suspended graphene devices. (a) Sample schematic. Few layer graphene ribbon (3 -10 layers thick) is suspended over a $1.4 \mu \mathrm{m} \times 0.2 \mu \mathrm{m}$ slit in a $100 \mathrm{~nm}$ thick silicon nitride (SiN) membrane (membrane size $\sim 40 \mu \mathrm{m} \times 40 \mu \mathrm{m}$ ). Inset: current-voltage characteristic of an as-fabricated nanoribbon, acquired in situ. (b) TEM image of a suspended graphene nanoribbon. Arrows indicate the edges of the graphene.

In the work [18] was demonstrated the possibility high-resolution modification of suspended multi-layer graphene sheets by controlled exposure to the focused electron beam of a transmission electron microscope. It was shown that this technique can be used to realize, on timescales of a few seconds, a variety of features, including nanometer-scale pores, slits, and gaps that are stable and do not evolve over time. Despite the extreme thinness of the suspended graphene sheets, extensive removal of material to produce the desired feature geometries is found to not introduce long-range distortion of the suspended sheet structure. Some results of these impressive experiments are given in figures 3 and 4 . 


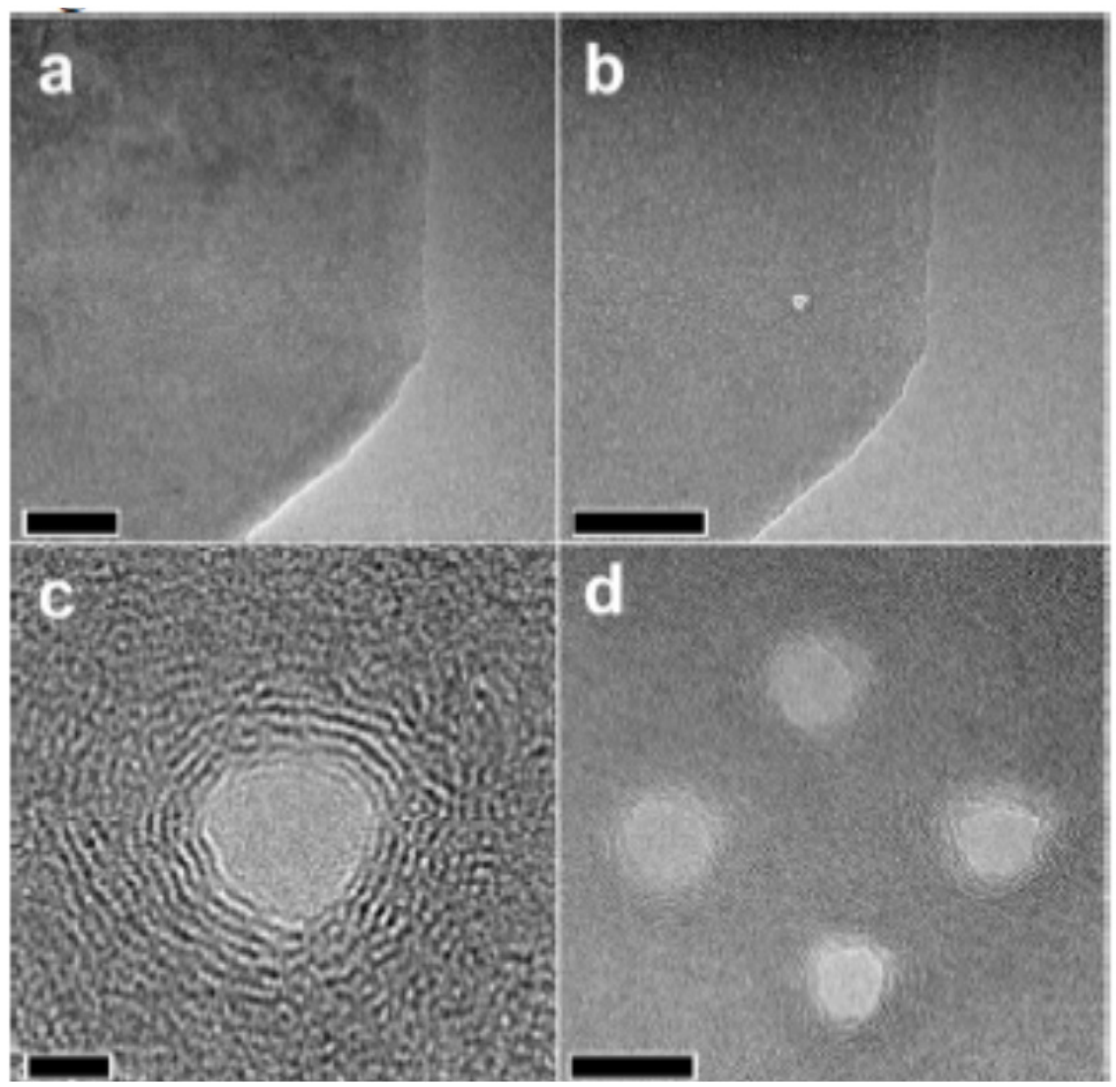

Figure 3. 18]. TEM images of a suspended graphene sheet is shown (a) before and (b) after a nanopore is made by electron beam ablation. (c) Higher magnification image of the nanopore. (d) Multiple nanopores made in close proximity to each other. (Scale bars are 50, 50, 2, $10 \mathrm{~nm}$ ). 


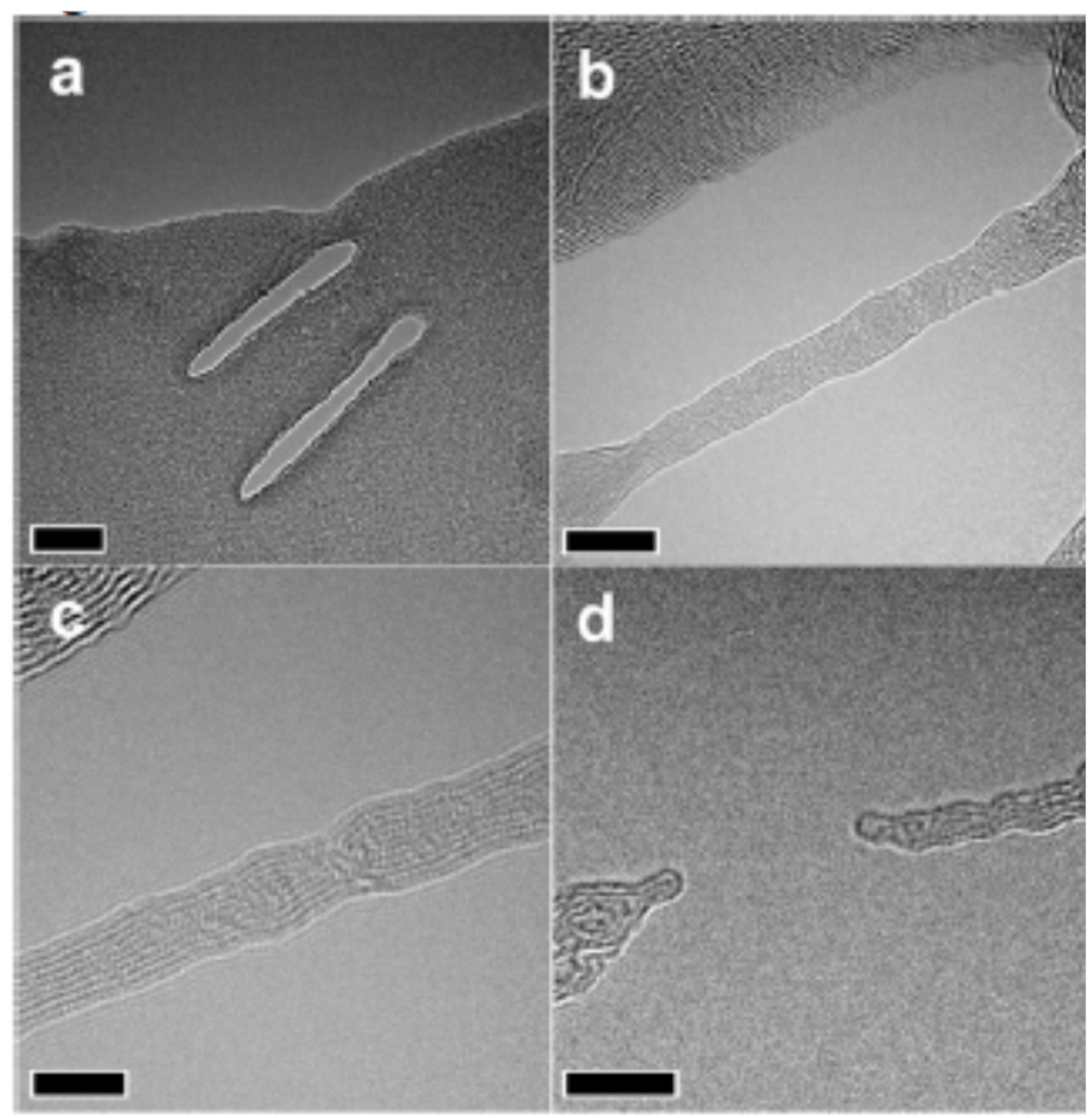

Figure 4. [18]. (a) Two $\sim 6 \mathrm{~nm}$ lines cut into a graphene sheet. (b) Electron irradiation is continued to create a $\sim 5 \mathrm{~nm}$ wide bridge. (c) Higher resolution of the bridge shows clear atomic order. (d) Small gap opened in the nanobridge by additional electron irradiation. Note that the cut ends are closed. (Scale bars are $20,10,5,5 \mathrm{~nm})$.

\section{Author details}

\section{V.E. Ptitsin}

Institute for Analytical Instrumentation of the Russian Academy of Sciences, Saint-Petersburg, Russia 


\section{Acknowledgement}

The work has been supported by the Russian Academy of Sciences and the Russian Foundation for Basic Research.

\section{References}

[1] J. Ready, Effect of High-Power Laser Radiation, Ed. S. I. Anisimov (Mir, Moscow, 1974) $468 \mathrm{p}$.

[2] G. A. Mesyts and D. I. Proskurovsky, Pulsed Electric Dicharge in Vacuum (Springer, Heidelberg, 1989) $256 \mathrm{p}$.

[3] K.A. Valiev, Physics of the Submicron Lithography (Nauka, Moscow, 1990) 528 p.

[4] Yu. A. Bykovsky, V. N. Nevolin, Laser Mass Spectrometry (Energoatomizdat, Moscow, 1985) $128 \mathrm{p}$.

[5] S. D. Kovaleski, R. M. Gilgenbach, L. K. Ang, Y. Y. Lau and J. S. Lash, Appl. Surf. Sci. 127-129, 947 (1998).

[6] J. Ziman, Electrons and Phonons (Mir, Moscow, 1962) 354 p.

[7] M. I. Kaganov, I. M. Lifchits and L.V. Tanatarov, ZhETF, 232 (1956).

[8] V. E. Ptitsin, Doctorate Thesis (Phys. \&Math.), IaI RAS, (1996).

[9] V. E. Ptitsin, Rev. Sci. Instr., 65, No. 4, 1476 (1994).

[10] Yu. V. Martynenko and Yu. N. Yavlinsky, Moscow Nuclear Power Institute Preprint, No. 4084/11, Moscow, (1985).

[11] V. E. Ptitsin, Phys. Status Solidi C 9, No. 1, 15-18 (2012)

[12] G. Muller, C. Schulteiss, Proc. 10th Int. Conf. on High Power Particle Beams (BEAMS94), 1994. pp. 833-836.

[13] W. Frey, C. Schulteiss, H. Bluhm, Proc. 14th Int.Conf. on High Power Particle Beams (BEAMS-2002), 2002. pp. 87-90.

[14] V.E. Ptitsin, V.F. Tregubov, Nauchnoije Priborostroenije, (Science, Saint- Petersburg), vol. 12, No 3, pp. $15-25$, (2002)

[15] V.E. Ptitsin, V.F. Tregubov, Ultramicroscopy, v. 95, pp. 131-138 (2003).

[16] S. D. Kovaleski, R. M. Gilgenbach, L. K. Ang, and Y. Y. Lau, Appl. Phys. Lett., vol. 73, No. 18, pp. 2576-2578 (1998).

[17] Michael D. Fischbein and Marija Drndic, Sub-10 nm Device Fabrication in a Transmission Electron Microscope, Nano Letters 7 (5), 1329-1337, (2007).

[18] Michael D. Fischbein and Marija Drndic, Electron beam nanosculpting of suspended graphene sheets, Applied Physics Letters 93, 113107, (2008).

[19] T. Gnanavel, Z. Saghi, M A Mat. Yajid, Y. Peng, B. J. Inkson, M. R .J. Gibbs and G. Mobus, Journal of Physics: Conf. Ser. 241 (2010) 012075.

[20] Ye Lu, Christopher A. Merchant, Marija Drndic, A.T. Charlie Johnson, In Situ Electronic Characterization of Graphene Nanoconstrictions Fabricated in a Transmission Electron Microscope, Nano Letters, 11 (12) 5184-5188, (2011).

[21] Neocera Company; see: www.neocera.com 
[22] Christopher A. Merchant, Ken Healy, Meni Wanunu, Vishva Ray, Neil Peterman, John Bartel, Michael D. Fischbein, Kimberly Venta, Zhengtang Luo, A. T. Charlie Johnson, Marija Drndic, DNA translocation through graphene nanopores, Nano Letters, 10 (8), pp 2915-2921, 2010. 